\title{
DETERMINATION OF RADIOACTIVELY LABELED GLOBULIN TURNOVER BY THE DIRECT WHOLE-BODY COUNTING TECHNIQUE *
}

\author{
By STUART W. LIPPINCOTT, STANTON H. COHN, HELEN HAMEL, \\ SAMUEL FINE AND SAMUEL KORMAN $\dagger$
}

(From the Medical Department, Brookhaven National Laboratory, Upton, N. Y.)

(Submitted for publication August 16, 1960; accepted December 16, 1960)

In clinical studies of the metabolic behavior of radioactively labeled proteins, the turnover (biological half-life or degradation rate) is usually determined from serum (or plasma) concentration curves which entail multiple blood sampling. The amounts of retained isotope are customarily inferred (by difference) from the analyses of excreta obtained from complete 24-hour samples collected for a number of successive days. In the present investigation the turnover of $\mathrm{I}^{131}$-labeled beta and gamma-globulins prepared by chemical fractionation was determined not only by the conventional method of blood and urine sampling but also by a new technique utilizing the whole-body gamma spectrometer. This device permits in vivo measurement of very low levels of an internally deposited gamma emitter (in this case, the $\mathrm{I}^{131}$ ). The procedure provides an excellent method for measuring retention of labeled globulins over long periods of time following administration of very low levels of isotopes. It also eliminates the tedious radiochemical analyses of excreta.

\section{PATIENTS AND METHODS}

Preparation of $I^{131}$-labeled globulins. The globulins used for the turnover studies were prepared from individual serums of 3 subjects. The first preparation was a $\gamma_{2}$-globulin fractionated by the method of Nichol and Deutsch (1). The donor was a 38 year old asymptomatic male who, after hospitalization and clinical investigation, was considered a suitable healthy subject. The second preparation was also a $\gamma_{2}$-globulin fractionated as noted above, but the donor was a patient with an established diagnosis of multiple myeloma, whose paper electrophoretic pattern disclosed a pronounced gamma peak. The third preparation, to be described elsewhere, was a beta globulin from a donor with an established diagnosis of multiple myeloma, whose electrophoretic

* This research was supported by the U. S. Atomic Energy Commission.

† Research Collaborator from the Montefiore Hospital, New York, N. Y. pattern showed a distinct beta peak and no gamma globulin.

The homogeneity of the fractionated pulins was studied by two physicochemical procedures. The electrophoretic characterization was carried out in the Spinco model of the Tiselius apparatus and the ultracentrifugal patterns of these same fractions were determined in the Spinco ultracentrifuge. In Figure 1 are shown the paper electrophoretic (a), Tiselius (b), and ultracentrifugation (c and d) determinations of the $\gamma_{2-}$ globulin fractionated from the sera of the normal healthy donor, Sm. There is a single peak in the paper strip and in both the ascending and descending limbs of the Tiselius electrophoretic patterns, indicative of a high degree of homogeneity in this $\gamma_{2}$-globulin. The sedimentation constant in (c) for the large peak was calculated as $\mathrm{S}_{20}$, giving a number of 6.48 , corresponding to a molecular weight of about 160,000 , which is in the accepted range for gamma globulin. It is to be noted that there is also seen in (c) another small peak representing a non- $\gamma_{\text {:- }}$ globulin not separated from the major fraction by the technique employed. The $S_{20}$ number for this peak was 9.95. In (d) the same preparation as in (c) was run after radioactive iodination but at a lesser concentration, thus showing a smaller peak. The $\mathrm{S}_{20}$ number for this peak was calculated to be 6.64 . The result of this determination was considered an indication that by this analysis the iodination with $\mathrm{I}^{131}$ did not show recognizable denaturation of the $\gamma_{2}$-globulin. However, it must be noted that the techniques employed do not necessarily show subtler degrees of degradation, as might conceivably be detected by chromatographic analyses of protein with mapping of the location of radioactivity. Figure 2 shows a study carried out in a way similar to that shown in Figure 1 but in this instance using the $\gamma_{2}$-globulin fractionated from the serum of hypergammaglobulinemic multiple myeloma donor patient $L$. In this preparation, the $S_{20}$ number for the major peaks was 6.60 in (c) and 6.64 in (d) after radioactive iodination. For the small contaminating peak in (c), the number was 9.80 .

Iodination, assay and calculations. The globulin preparations were iodinated and their radioactive assays determined as described before for serum and urine concentration curves (2). This procedure was performed in a well-type scintillation counter using standard electronic components. The number of microcuries in the labeled globulins ranged from 17 to $50.16 \mu \mathrm{c}$ and the 

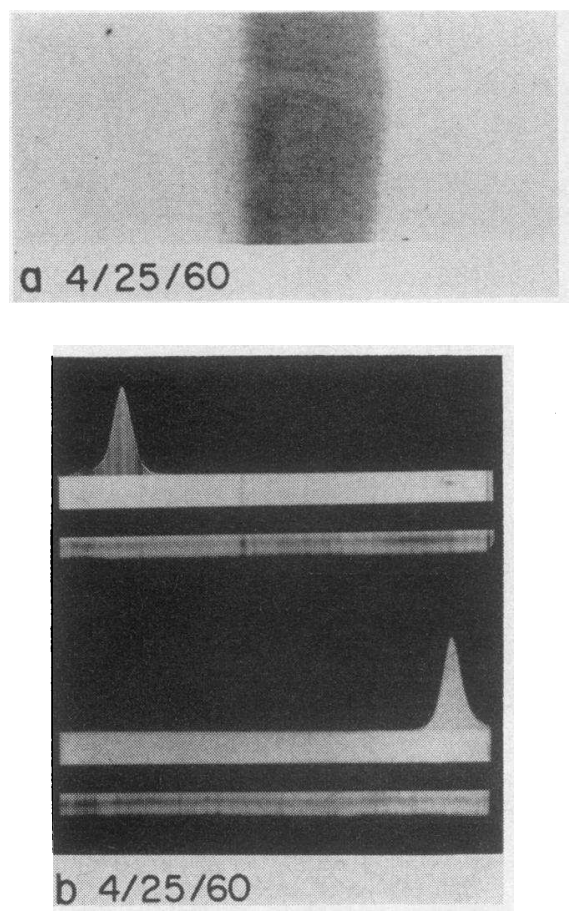

Fig. 1. Paper electrophoretic (a), Tiselius (b) and Ultracentrifugation (c, Before and d, AFTER IODINATION) DETERMINATIONS OF $\gamma_{2}$-GLOBULIN FRACTIONATED FROM SERUM OF NORMAL DONOR Sm.
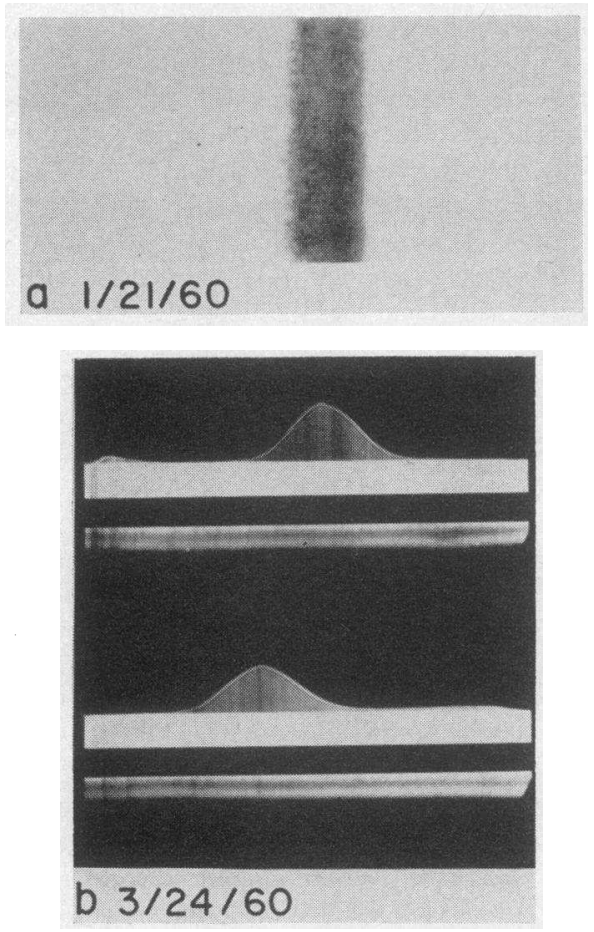

Fig. 2. Paper electrophoretic (a), Tiselius (b) and ultracentrifugation (c, Before and d, AFTER IODINATION) DETERMINATIONS OF $\gamma_{2}$-GLOBULIN FRACTIONATED FROM SERUM OF HYPERGAMMAGLOBULINEMIC MULTIPLE MYELOMA DONOR PATIENT L.

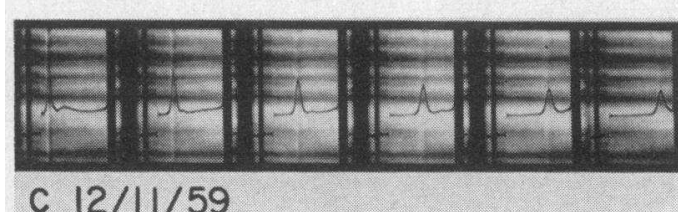

\section{c $12 / 11 / 59$}

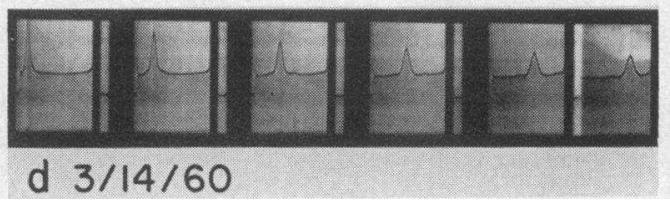




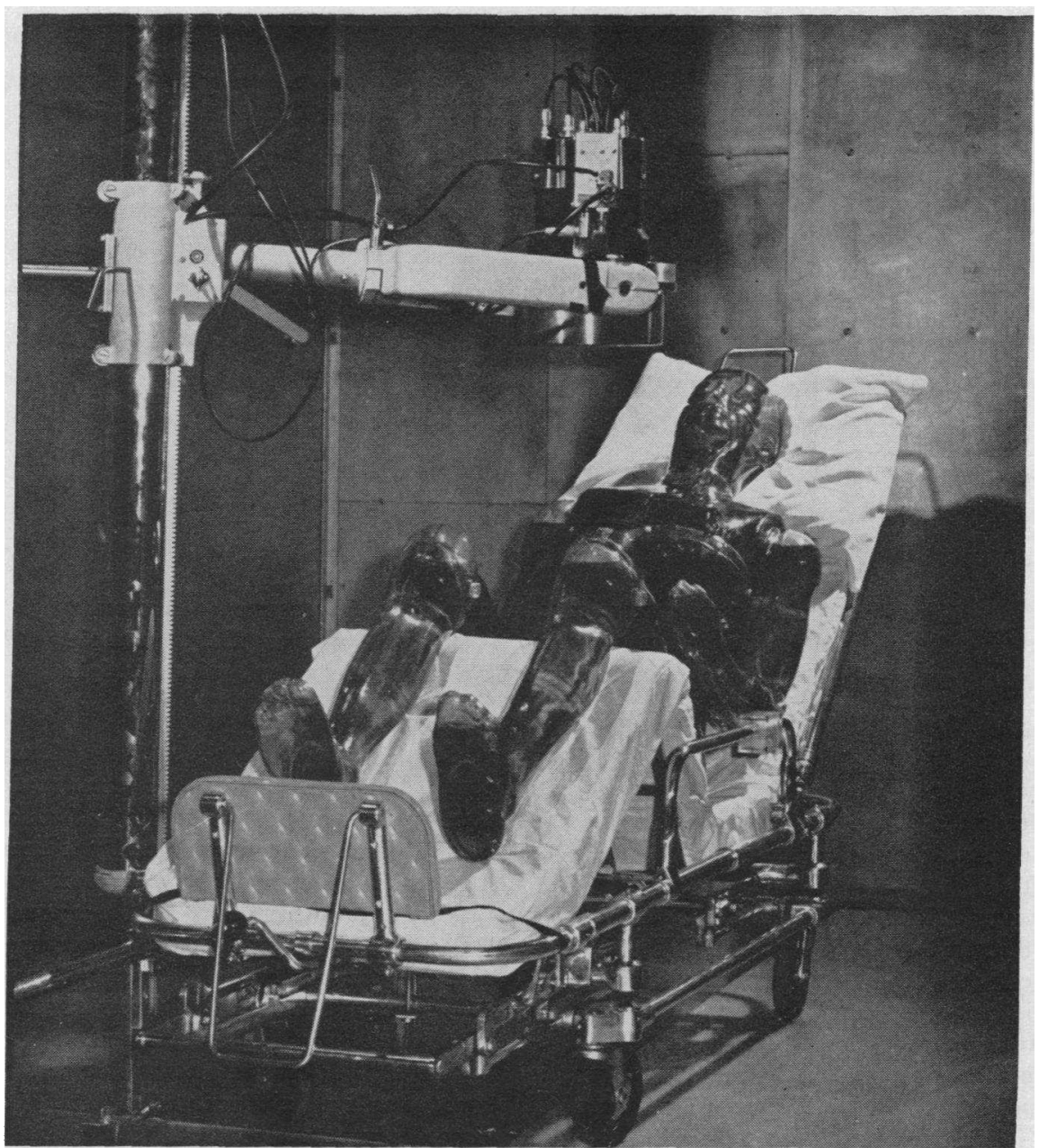

Fig. 3. Plastic calibration phantom in standard counting position in steel. ROOM.

amount of protein from 2.5 to $4.9 \mathrm{mg}$. The calculations for determining the turnover consisted in plotting the radioactivity (after correction for physical decay) as a function of the time of sampling on semilogarithmic paper. The rapid early decay of the curve is thought to be due to distribution and mixing of the injected labeled preparation, while the second part of the curve is designated as the slow component. It is an exponential decay indicated by the straight line and is considered to represent the rate of degradation of the injected labeled protein (3).

Patients. The diagnoses of multiple myeloma in the two donors with this disease and in the recipient in whom the turnover studies were carried out were established by microscopic examination of bone marrow aspirations. Chemical determinations of the serum proteins, paper electrophoretic serum patterns and X-ray bone surveys also supported the diagnoses. In the case of the recipient, Lugol's solution was administered 3 days before the injection of the labeled globulin and daily throughout the study to prevent thyroid accumu- lation of radioactive iodine. The maximal radiation dosage to the recipient was calculated to be $0.013 \mathrm{rad}$ in a $50 \mathrm{~kg}$ patient, assuming an effective half-life of 4.3 days and taking the blood as the critical organ (2).

Whole-body counter. A whole-body gamma scintillation spectrometer was utilized in this study for the in vivo detection of gamma radiation. The high quantum efficiency for gamma makes this instrument very valuable for measuring small tracer amounts of radioactive isotopes. The gamma radiation from $\mathrm{I}^{131}$-labeled globulin retained in the body as a function of time was measured with an energy-sensitive $\mathrm{NaI}$ crystal detector. The data from the spectrometer are in the form of pulse-height spectra obtained by analysis of the amplified output of the detector. The information plotted is the counting rate per unit energy interval.

The fact that the output pulse generated by the scintillation detector is proportional to the energy dissipated in the scintillator by the gamma photon gives this counter its greatest advantage. $\mathrm{I}^{131}$ in this study was identified by its distinctive energy spectrum, and the 


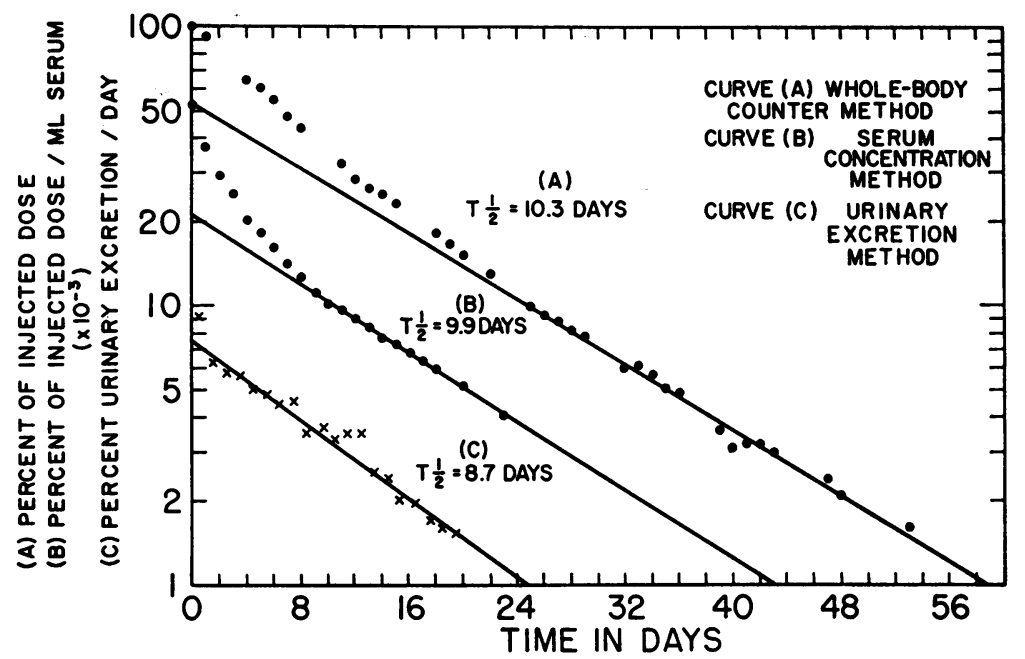

Fig. 4. WhOLE-BODY GAMMA SPECTROMETER METHOD VERSUS CONVENTIONAL SERUM CONCENTRATION AND URINARY METHODS. Turnover of $\mathrm{I}^{131}$ multiple myeloma beta globulin in a hypergammaglobulinemic patient with multiple myeloma.

number of pulses in its $0.364 \mathrm{Mev}$ photopeak was taken as the quantitative measure of the amount present. Corrections were made for the appropriate background of the patient obtained before the injection of each labeled compound.

The Brookhaven National Laboratory whole-body counter is built around a $4 \times 8$ inch NaI (T1) crystal detector mounted with three 3 -inch photomultiplier tubes. This detector is connected to a linear amplifier and by appropriate circuitry to a Penco (model PA-4) 100channel pulse-height analyzer. The crystal detector is located in a shielded room, $6 \times 7 \times 9$ feet, constructed of 6 inch steel. The interior of this 42 ton room is lined with lead, cadmium and copper for shielding against the low energy components of the background radiation. The heavy shielding effectively reduces the gamma radiation to the detector from both cosmic rays and gammaemitting contaminants in building materials, and increases the signal-to-noise ratio for the system.

The patient is counted in a chair placed in a standard position under the crystal as shown in Figure 3. The crystal is mounted 19 inches above the apex of the chair so that it "sees" a large fraction of the whole body. This counting position approaches optimal geometry while at the same time assuring to the patient a position which is both comfortable and easily reproducible. The patient was counted immediately before and after i.v. administration of the labeled compound and counts were made five times weekly over a period of approximately 2 months for each study, each count taking 10 minutes.

The stability of the counting efficiency of the wholebody counter is quite high. The percentage deviation of the counting efficiency in terms of counts per minute per microcurie of $\mathrm{Cs}^{137}$ in the $0.66 \mathrm{Mev}$ photopeak over the course of the study was 0.24 per cent. At the same time the fluctuation in total background ( 0.1 to $2 \mathrm{Mev})$ in a 10-minute count was 1.1 per cent. The counting efficiency of the counter for the $\mathrm{I}^{131}$ photopeak is 0.24 per cent. The precision of the counter for a 10-minute count for $\mathrm{I}^{131}$ in the patient was $7.3 \mathrm{~m} \mu \mathrm{c}$. The counting rate response of the counter to $\mathrm{I}^{131}$ is linear up to $100 \mu \mathrm{c}$. The error in reproducing the geometry of the patient in the chair is 1.8 per cent. At 60 days after the administration of $50 \mu \mathrm{c}$ to patients, the error in the counting rate was 1.2 per cent. Following the administration of $17 \mu \mathrm{c}$ to 1 patient with a faster turnover, the counting error at 49 days was 12.3 per cent.

The 100 per cent retention value was taken at $30 \mathrm{~min}$ utes after i.v. injection. The whole-body $\mathrm{I}^{131}$ activity drops only a few per cent in the first few hours, then rises at about 6 hours, after which it falls off to the end of the experiment. One of the difficulties encountered in whole-body counting of patients immediately after i.v. administration of isotopes arises from the initial distribution of the isotope as a function of time. This difficulty is greatest within the first few hours or days, depending on the localization of the radioelement or labeled compound in the organs of ultimate deposition. It is this rapidly changing distribution of the isotope that accounts for the anomalous rise in $\mathrm{I}^{131}$ activity at 6 hours. The apparent rise in activity (which may result from the label being fixed in the thyroid or gastric glands) reflects the altered geometry with respect to the relative position of the isotope to the detector. This problem will be discussed in detail in another paper. In this study the early redistribution of the isotope is not a problem since the retention, as measured by wholebody counting and by cumulative excretion data, correlates very closely from 3 days to the end of the study.

\section{RESULTS}

The turnover of a beta globulin prepared from the serum of a multiple myeloma patient and la- 
beled with $I^{131}$ is shown in Figure 4 . The recipient in whom the turnover study was performed was a hypergammaglobulinemic patient with multiple myeloma. In curve $B$ the half-time $\left(\mathrm{T}_{\frac{1}{2}}\right)$ was determined by the serum concentration method as being 9.9 days and by the urinary excretion method as 8.7 days (curve $\mathrm{C}$ ). These values are in good agreement and were established by two conventional methods. Throughout the period of these two studies and continuing to the fifty-third day, the patient was placed in the whole-body counter 34 times and the decrease in the radioactivity was determined with progression of time. The $T_{\frac{1}{2}}$ established by the wholebody counter method was 10.3 days. The values obtained in curves $\mathrm{A}$ and $\mathrm{B}$ are essentially the same, while that from $C$ is slightly shorter. This is not unusual for the urinary excretion curve, since it is always possible that a specimen may be lost and because a very small amount of the label appears in the feces and is therefore not accounted for.

In the same recipient for whom the data are shown in Figure 4 and at a subsequent time, this patient was injected with $\mathrm{I}^{131}-\gamma_{2}$-globulin prepared from the serum of a hypergammaglobulinemic multiple myeloma patient. In this instance (Figure 5) the $T_{\frac{1}{2}}$ determined from the urinary excretion data and that from the whole-body counter technique were compared. Instead of counting

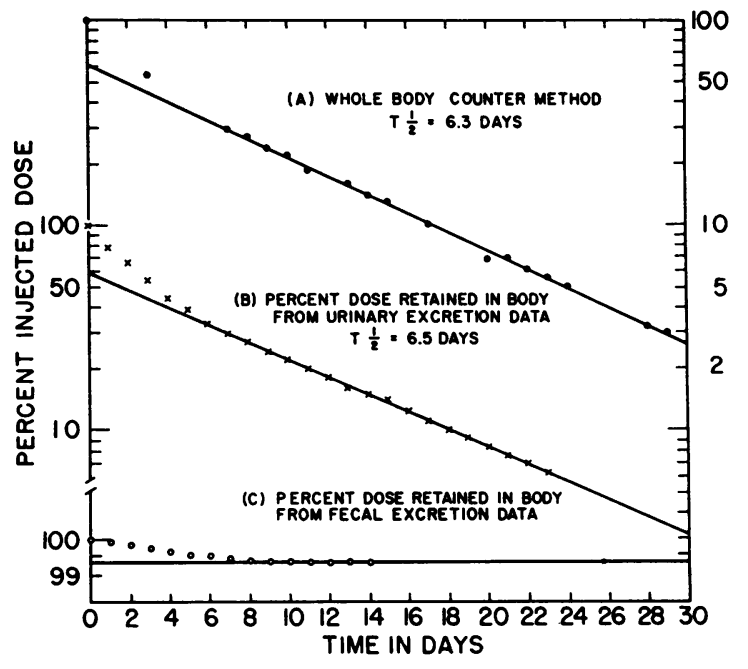

Fig. 5. TURNover of I $^{131}$-LABELED MULTIPLE Myeloma

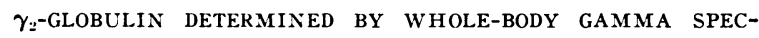
TROMETER AND URINARY EXCRETION METHODS IN A HYPERgammaglobulinemic multiple myeloma patient.

an aliquot of a 24-hour urine collection in a scintillation well counter, the entire 24-hour urine collection obtained on each of 23 successive days was counted in the whole-body counter.

The percentage dose retained in the body was then calculated from the gamma spectrometer urinary excretion data and the $T_{\frac{1}{2}}$ determined. The $\mathrm{T}_{\frac{1}{2}}$ obtained by the whole-body counter method was 6.3 days and for the data from urinary excretion (percentage dose retained) was 6.5 days. During this study, 24-hour fecal collections were

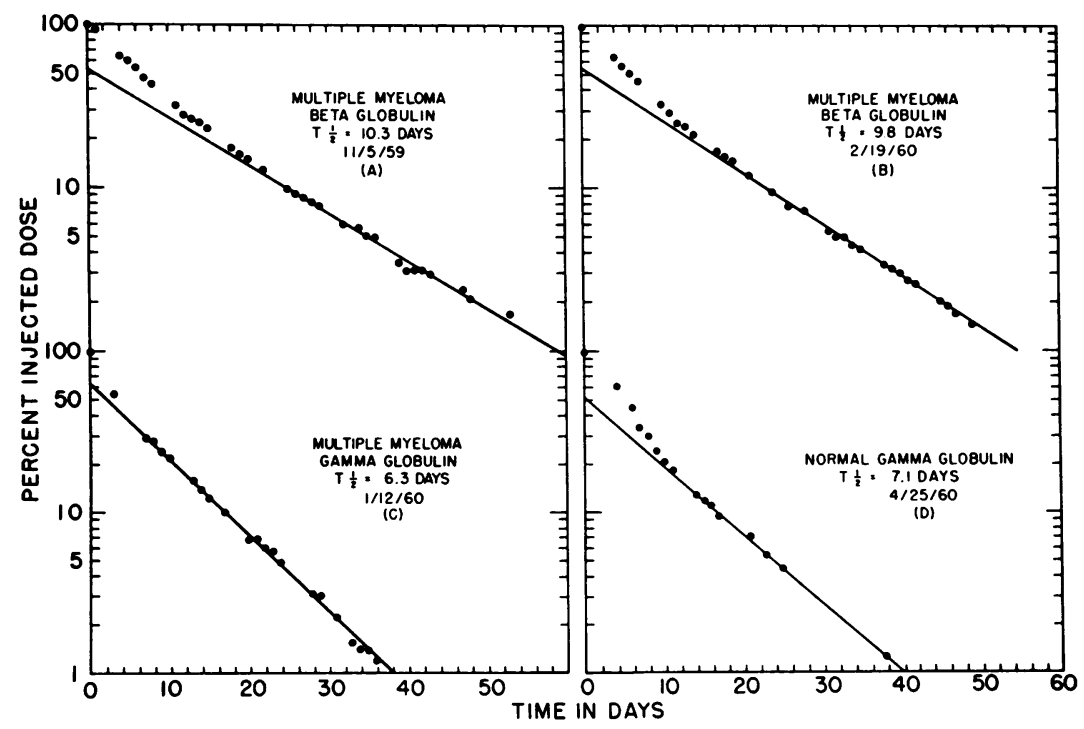

Fig. 6. Comparisons of turnovers of various I ${ }^{131}$-LABeled Proteins DETERMINED BY WHOLE-BODY GAMMA SPECTROMETER METHOD IN A HYPERGAMMAGLOBULINEMIC PATIENT WITH MULTIPLE MYELOMA, 
made for 14 days and each entire collection was counted in the whole-body device to determine the amount of loss of the radioactive label appearing in the feces. It can be seen that it did not exceed 1 per cent.

Replicate testing cannot be carried out with these procedures, but the accuracy of the wholebody counter method was determined as shown in Figure 6. Radioactively labeled beta globulin was injected into the patient as indicated in curve $\mathrm{A}$ and the $\mathrm{T}_{1}$ was 10.3 days. About 3.5 months later, beta globulin was again labeled, injected into the same patient, and the $T_{1}$ was 9.8 days as seen in curve B. Between those two studies multiple myeloma gamma globulin was labeled, injected into the same patient, and the $T_{1}$ was 6.3 days (curve $\mathrm{C}$ ). About 3.5 months after this, the $\mathrm{T}_{1}$ for labeled normal gamma globulin was determined and found to be 7 days (curve D). Thus, in a period of about 7 months four turnover studies were carried out by the whole-body counter technique in the same patient, and the reliability of the method, based on reproducibility, was established for the two types of globulins.

\section{DISCUSSION}

The validity of the turnover values obtained in this study is dependent upon several factors. The protein preparations used must meet a rigid standard of homogeneity, for at best globulins will not be as homogeneous as albumin. By determining the electrophoretic and ultracentrifugal patterns, suitable products can be selected for labeling. Radioactive labeling should not degrade the protein. This was investigated by ultracentrifugal analysis before and after iodination and found to be satisfactory, although it is recognized, as noted previously, that a more precise method might better be employed. A further consideration concerning the proteins studied is concerned with resultant daily degradation rate $\left(1001 \mathrm{n} 2 / \mathrm{T}_{\frac{1}{2}}\right)$. For the beta globulins from the serum concentration curve, this rate was 7.0 per cent per day and from the urinary excretion data (urinary excretion per day/ dose retained) it was 7.4 per cent per day (Figure 4). The degradation rate for the gamma globulin determined from the whole-body counter method was 11.0 per cent per day and from the urinary excretion data it was 11.6 per cent per day (Figure 5).
To be of value, the whole-body in vivo gamma spectrometer technique results must compare favorably with those obtained by calculation from serum and urinary excretion radioactivity concentrations. This was demonstrated for both beta and gamma globulin turnovers and in a separate study (unpublished data) for albumin in which the advantages of the whole-body counting method were found to be: 1) elimination of serial blood sampling and collection of excreta for radiochemical analyses ; 2) direct determination of body content rather than estimation by difference or extrapolation from fractional measurement; and 3) utilization for tracer studies of very low levels of an internally deposited gamma emitter over long periods of time.

\section{SUMMARY}

The turnover rates of radioactively labeled beta and gamma globulins in a hypergammaglobulinemic multiple myeloma patient were compared by the conventional serum and urinary excretion methods with those found using the in vivo whole-body gamma spectrometer counting method. The fractionated radioactively labeled globulins used in these studies were considered by free (Tiselius) electrophoresis and ultracentrifugal patterns to have an acceptable degree of homogeneity. The half-life for the beta globulin was 9.9 days by the serum concentration method, 8.7 days by the urinary excretion method and in two different studies by the in rivo whole-body counter method, 10.3 and 9.8 days, respectively. The half-life for the gamma globulin utilizing urinary data was 6.5 days as compared to 6.3 days for the whole-body counter method. By the latter technique, with a different gamma globulin preparation, the halflife was 7.1 days.

\section{REFERENCES}

1. Nichol, J. C., and Deutsch, H. F. Biophyiscal studies of blood plasma proteins. VII. Separation of $\gamma$-globulin from the sera of various animals. J. Amer. chem. Soc. 1948, 70, 80.

2. Lippincott, S. W., Korman, S., Fong, C., Stickley, E., Wolins, W., and Hughes, W. L. Turnover of labeled normal gamma globulin in multiple myeloma. J. clin. Invest. 1960, 39, 565.

3. Sterling, $K$. The turnover rate of serum albumin in man as measured by $\mathrm{I}^{131}$-tagged albumin. J. clin. Invest. $1951,30,1228$. 